\title{
Acknowledgment to JCMK Editorial Board and Peer-Reviewers for contribution in 2018
}

\author{
Laura Dybyssova \\ Executive Secretary, on behalf of editorial team of Journal of Clinical Medicine of Kazakhstan
}

On behalf of Journal of Clinical Medicine of Kazakhstan, we would like to express our appreciation to all editorial and advisory board members, reviewers and authors who contributed to this journal in year 2018.

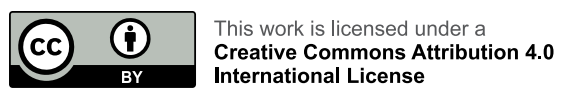

J Clin Med Kaz 2019;1(51):6-7

\section{EDITORIAL AND ADVISORY BOARD MEMBERS IN 2018}

\begin{tabular}{|c|c|}
\hline $\begin{array}{l}\text { Editor-In-Chief } \\
\text { Abay Baigenzhin (Kazakhstan) } \\
\text { Editors } \\
\text { Temirlan Karibekov (Kazakhstan) } \\
\text { Saltanat Tuganbekova (Kazakhstan) } \\
\text { Associate Editor } \\
\text { Abduzhappar Gaipov (Kazakhstan) } \\
\text { Executive Secretary } \\
\text { Laura Dybyssova (Kazakhstan) } \\
\text { Editorial Board } \\
\text { Manarbek Askarov (Kazakhstan) } \\
\text { Zulfiya Orynbayeva (USA) } \\
\text { Rimantas Benetis (Lithuania) } \\
\text { Galina Fedotovskikh (Kazakhstan) } \\
\text { Ospan Mynbaev (Russian Federation) } \\
\text { Gunay Akbarova (Azerbaijan) } \\
\text { Selman Unverdi (Turkey) } \\
\text { Ulan Kabayev (Kazakhstan) } \\
\text { Talgat Nurgozhin (Kazakhstan) } \\
\text { Yuriy Kazakov (Ukraine) } \\
\text { Almaz Makenjan uulu (Kyrgyz Republic) } \\
\text { Pakhongir Alidjanov (Uzbekistan) } \\
\text { Oybek Rustamov (Australia) }\end{array}$ & $\begin{array}{l}\text { International Advisory Board } \\
\text { Turgut Teke (Turkey) } \\
\text { Kubes Jiri (Czech Republic) } \\
\text { Yaroslav Tolstyak (Ukraine) } \\
\text { Rustam Mustafin (Bashkortostan, } \\
\text { Russian Federation) } \\
\text { Adem Kucuk (Turkey) } \\
\text { Yana Sotskaya (Ukraine) } \\
\text { Ainura Dzhangaziyeva (Kyrgyz Republic) } \\
\text { Mehtap Tinazli (Turkey) } \\
\text { Yulia Lunitsyna (Russian Federation) } \\
\text { Yuksel Ersoy (Turkey) } \\
\text { Rikhsi Sabirova (Uzbekistan) } \\
\text { Nurdin Mamanov (Kyrgyz Republic) } \\
\text { Mariya Derbak (Ukraine) } \\
\text { National Advisory Board } \\
\text { Suyundik Imankulov (Kazakhstan) } \\
\text { Anatoliy Kolos (Kazakhstan) } \\
\text { Vitaliy Koikov (Kazakhstan) } \\
\text { Saule Abseitova (Kazakhstan) } \\
\text { Almagul Kushugulova (Kazakhstan) } \\
\text { Marlen Doskali (Kazakhstan) } \\
\text { Kakharman Yesmembetov (Kazakhstan) } \\
\text { Nelya Bissenova (Kazakhstan) } \\
\text { Gauri Bapayeva (Kazakhstan) } \\
\text { Ibragim Tashev (Kazakhstan) } \\
\text { Bagdat Imasheva (Kazakhstan) } \\
\text { Galiya Shaimardanova (Kazakhstan) } \\
\text { Nasrulla Shanazarov (Kazakhstan) } \\
\text { Adilzhan Albazarov (Kazakhstan) } \\
\text { Elmira Chuvakova (Kazakhstan) } \\
\text { Zhannat Taubaldiyeva (Kazakhstan) } \\
\text { Aidos Konkayev (Kazakhstan) } \\
\text { Samat Saparbayev (Kazakhstan) } \\
\text { Olga Ulyanova (Kazakhstan) } \\
\text { Galiya Orazova (Kazakhstan) } \\
\text { Manana }\end{array}$ \\
\hline
\end{tabular}




\section{PEER-REVIEWERS WHO CONTRIBUTED TO THIS JOURNAL DURING 2018}

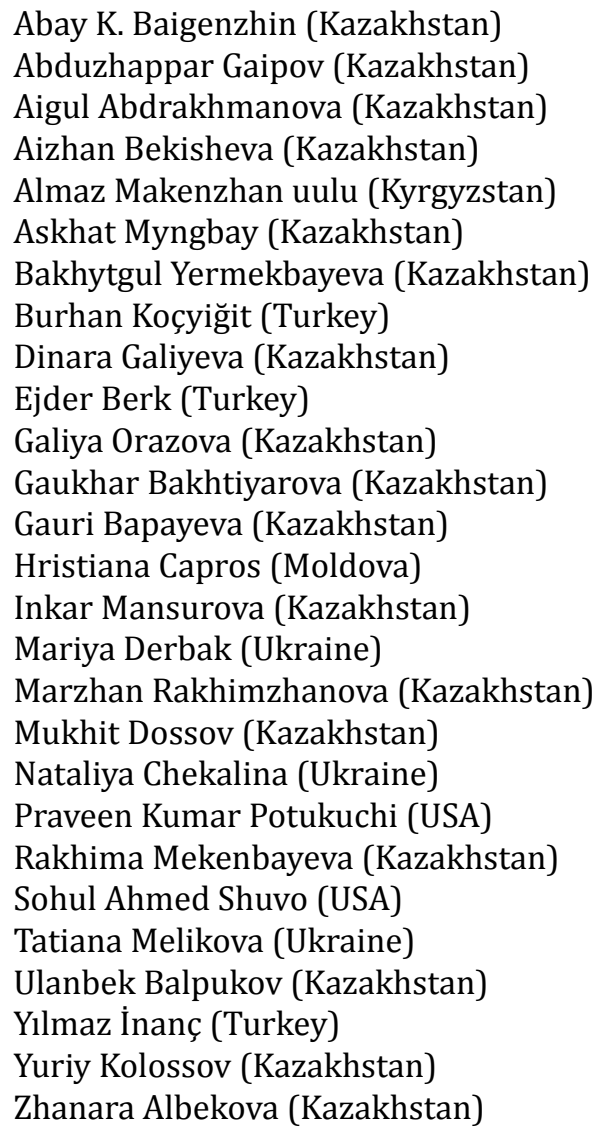

\section{OUR STATISTICS}

Journal of Clinical Medicine of Kazakhstan published 4 regular issues and 1 supplement issue in 2018

- Volume 4, Number 50 (2018) with 7 articles

- Volume 3, Number 49 (2018) with 9 articles

- Volume 2, Number 48 (supplement 1) (2018) with 51 articles

- Volume 2, Number 48 (2018) with 11 articles

- Volume 1, Number 47 (2018) with 10 articles

During 2018, 15 articles were rejected, rejection rate was $40 \%$ (considering articles from regular issues).

\section{AUTHORS 2018}

Authors and coauthors who contributed to this journal in 2018 were from the following countries: Kazakhstan (dominantly), Russia, Ukraine, Turkey, Belarus, Oman, Uzbekistan, Tajikistan, Azerbaijan, United Kingdom, USA, Nigeria, Indonesia, Kyrgyzstan, Korea.

The editorial team of Journal of Clinical Medicine of Kazakhstan would like to express gratitude for your valuable support and being part of our excellent team. We appreciate your continuous efforts and hope to continue receiving your great feedback, valuable ideas, and interesting scientific papers to further improve the quality and impact of the Journal of Clinical Medicine of Kazakhstan.

Sincerely yours,

Editorial Office 